\title{
Corticosteroids in Leprosy A Guide to their Systemic Use
}

S. G. BROWNE, M.D., F.R.C.P., F.R.C.S., D.T.M.

I eprosy Service Research Unit, Uzuakoli, Eastern Nigeria

This article, written at the Editor's request, aims at providing practical help to leprosy workers who may be confused by the apparently conflicting advice tendered by leprologists in different countries and the competing claims of drug manufacturers. It will not present a careful weighing of scientific arguments or experimental evidence, reinforced by numerous references to the literature, but will rather be a simple guide as to when and how to use adrenal corticosteroids and certain of their synthetic analogues that have within recent years been made available. Other conditions (such as dapsone dermatitis) that respond to corticosteroid therapy will not be dealt with.

\section{N D I C A T I O N S}

Corticosteroids are potent and valuable anti-inflammatory agents, and a personal acquaintance with their indications and limitations is essential for all who are responsible for the care of leprosy patients. While they are without effect on the underlying pathological processes of leprosy, they do control severe exacerbation and permit continuation or resumption of anti-leprosy therapy. They should not be used in the absence of definite indications, or without due regard to the dangers of prolonged administration. Thus, they should not be prescribed for slight degrees of erythema nodosum leprosum or for an exacerbation that is accompanied by minimal systemic disturbance. Nor should they be given to children except for very severe reaction. It is easy to begin corticosteroid therapy: it is difficult and sometimes impossible to stop. The practice of issuing corticosteroids for outpatient administration in reactional states in leprosy cannot be too strongly condemned. In the acute stages, the patient must be at bed rest, under strict medical supervision; in the subsequent subacute stage, he may be ambulant, but must still be under medical supervision.

Corticosteroids are indicated, in lepromatous leprosy:

I. When the systemic disturbance from acute exacerbation is severe from the outset, when the localized cutaneous signs of hypersensitivity (erythema nodosum leprosum) are widespread and recurrent and persistent, when lepromatous infiltration and discrete lesions are oedematous and hyperaemic, and especially when the eyes are affected. Severe systemic disturbance is indicated mainly by: raised temperature (i.e. above I $02{ }^{\circ} \mathrm{F}, 39^{\circ} \mathrm{C}$ ) and disabling malaise.

2. When the systemic disturbance and the local manifestations of reaction are moderately severe, but fail to respond to the methods of control habitually tried initially. If after a fortnight of such treatment, the 
patient (at bed rest, and no longer receiving anti-leprosy therapy) is unimproved, then recourse should be had to corticosteroids.

3. In severe peripheral neuritis of sudden onset and accompanied by marked local swelling and tenderness of nerve trunks at the sites of predilection.

4. When iritis or iridocylitis supervenes in a patient with lepromatous leprosy that is either mildly reacting or even perhaps otherwise clinically stable; in other words, the eye condition requires corticosteroids, whereas the general condition does not.

\section{CONTINUATION OF ANTI-LEPROSY THERAPY}

Opinions differ widely on this question: there are those who would suppress anti-leprosy drugs in all patients undergoing reaction, however mild, and at the other extreme there are some who advocate the continuation of therapy, at whatever cost.

In mild exacerbation, requiring nothing more than sedatives, treatment may be maintained without reducing the dose. In rather more severe cases, the dose should be halved and more energetic measures to combat the reaction should be instituted (anti-histaminics, anti-malarials, antimony). In moderately severe cases, anti-leprosy therapy should be suppressed forthwith. In patients requiring corticosteroids for severe exacerbation, it is advisable to discontinue anti-leprosy therapy temporarily, until the reaction is controlled, and then to resume with the same drug at half the preceding dose, under corticosteroid cover.

In some patients, it appears that resumption of anti-leprosy therapy is less likely to provoke a return of the exacerbation if the original drug is replaced by another, e.g. thiambutosine replacing dapsone.

\section{POSSIBLE INDICATIONS}

The action of corticosteroids in some conditions is problematical, and the advisability of their use both from the immediate and long-term standpoints is a matter on which opinions may legitimately differ. The purpose of systemic corticosteroid therapy here is to supplement non-specific local therapy, both chimiotherapeutic and physical, and to accelerate and enhance its effect. In the following conditions, a dramatically rapid local improvement may follow their use in some patients:

I. Reactional tuberculoid leprosy, where the numerous lesions are swollen, tense, hyperaemic and painful, and especially where superficial nerves share in the hypersensitive state.

2. Borderline leprosy in acute reaction, especially when accompanied by systemic disturbance and peripheral neuritis.

3. Extensive tuberculoid lesions on the face in reaction, especially when the lesions are in the neighbourhood of important nerves (e.g. branches of the facial, and less importantly, branches of the trigeminal). It is important to begin corticosteroid therapy early in such cases, if irreversible facial palsy is to be averted. 
4. Acute localized neuritis, of sudden onset, and often confined to a single nerve in the neighbourhood of a reacting tuberculoid lesion (e.g. near the elbow or knec), especially when accompanied by sudden paresis (e.g. of the intrinsic muscles of the hand, or drop foot). Here again, corticosteroid treatment must be instituted at once to forestall severe and permanent nerve damage.

5. Severe nasal obstruction of acute onset in lepromatous leprosy, duc to ocdema of the mucosa.

6. Acutely swollen, tense and painful ear-lobes.

PRODUC:TS REC:OMMENDED

Prednisolone is the best corticosteroid for general use in leprosy. Weight for weight, it is five times as potent as cortisone acetate, almost as potent as triamcinolone, and about a seventh as potent as betamethasone and dexamethasone. It is marketed in scored tablets usually containing 5 to $2 \frac{1}{2} \mathrm{mgm}$. of active product.

'The incidence of undesirable side-effects appears to vary but little between one proprictary preparation and another, whatever slight modifications in side-chains are made. A radical change in the molecule, however, will make for a definite difference in action. Triamcinolone has a decided advantage in possessing less tendency to provoke oedema from water- and salt-retention, and betamathasone and dexamethasone seem definitcly less apt to provoke side-effects than the older preparations.

The use of ACTH has certain theoretical advantages, notably that of ensuring a continued output of diverse corticosteroids from the patient's own adrenal glands, with resulting decreased risks of untoward effects during withdrawal. In practice, however, it is found that provided the dosage of prednisolone is within the limits here advocated and provided also that withdrawal is accomplished carefully and slowly (i.e. up to two or even three months after prolonged medication) ACTH has no advantages over prednisolone.

For rapid action, however, intravenous injection of ACTH in doses of I $0-25$ international units, repeated at daily intervals and followed by oral prednisolone, has its advocates.

Cortisone acetate by subcutaneous injection in daily doses of up to $25 \mathrm{mgm}$., brings a rapid effect to bear on the inflammatory condition. For the first acute attack of exacerbation, which has not responded to standard treatment, a simple and satisfactory régime consists of cortisone by injection, $3 \mathrm{ml}$. daily for 3 days, then $2 \mathrm{ml}$. daily for 3 days, and then $\mathrm{I} \mathrm{ml}$. daily for 3 days. This will frequently be successful in aborting the reaction. If, however, while the daily dosage of cortisone is being reduced, a worsening of the clinical state occurs, then oral prednisolone should be given in addition to the daily injections of cortisone continued as prescribed above. The dose of prednisolone should be just sufficient to control the acute signs and symptoms. Thus, if $3 \mathrm{ml}$. of cortisone acetate was sufficient, but I ml. proves inadequate, then the oral prednisolone supplement should 
begin with the equivalent of the difference between the two (i.e. $2 \mathrm{ml}$. or $50 \mathrm{mgm}$. of cortisone), which equals about $10 \mathrm{mgm}$. of prednisolone, given preferably in four doses of $2 \frac{1}{2} \mathrm{mgm}$. in 24 hours.

In severe first reactions, and in moderately severe recurrent reactions where the first reaction necessitated corticosteroid therapy, injectable cortisone should be given a 48 -hour trial at the daily dosage of $75 \mathrm{mgm}$. ( $3 \mathrm{ml}$.), but if the condition is still uncontrolled at this dose and the patient is scriously distressed, then there should be no hesitation in doubling the dose, and giving $50 \mathrm{mgm}$. or $2 \mathrm{ml}$. thrice daily for three days, reducing the dose gradually as usual. If the exacerbation is so severe as to demand corticosteroids, then these drugs are the only drugs that will control the exacerbation. In these cases, the withdrawal must be more gradual and more prolonged, i.e. reducing the dose by a half or a quarter at weekly intervals and ensuring (by giving divided doses) that there is an adequate blood level throughout the 24 hours. The dose need not be increased when only slight worsening of the reactional condition occurs.

\section{PROLONGED TREATMENT WITH CORTICOSTEROIDS}

The only justification for the prolonged administration of corticosteroids in persistent reactional states in lepromatous leprosy is that anti-leprosy therapy may be resumed or continued under their cover. If anti-leprosy drugs have been completely suppressed, then cautious resumption of therapy is advocated, i.e. at half or even a quarter of the dose that was given before the onset of the exacerbation.

If, however, prednisolone had to be introduced during or after the course of injectable cortisone, then it is advisable to control the symptoms with prednisolone, while anti-leprosy therapy is cautiously resumed, and then to reduce gradually the amount of prednisolone given till it is finally discontinued.

Some patients cannot be weaned from corticosteroids for many months despite repeated attempts. They may be acutely sensitive to very slight reductions in the maintenance dose. These cases will tax to the utmost the patience and resource of the medical attendant. The upper limit of safety for continuous prolonged corticosteroid medication is probably Io $\mathrm{mgm}$. of prednisolone daily for an adult weighing $120 \mathrm{lbs}$., or 65 kilos, with permissible temporary increases for break-through episodes of exacerbation. At this dose, adrenal function is probably not completely suppressed, pituitary function is undisturbed and potassium supplements are not required.

When lepromatous patients are in such an unstable hypersensitive immunological state that even minute doses of dapsone appear to precipitate further attacks of disabling reaction, it is advisable to institute a régime of very gradually increasing doses of a dilute solution of solapsone, given orally, as for desensitization for dapsone dermatitis, under continuing corticosteroid cover. In this way, a therapeutic dose of dapsone may be attained in from three to six months. Repeated attempts should 
then be made to wean the patient from corticosteroids. Pregnancy may provide an opportunity for such weaning.

The writer has seen lepromatous patients in persistent reaction achicve clinical cure and bacteriological negativity after five years of careful corticosteroid medication, apparently none the worse.

\section{E M E R G E N C I E S}

When patients taking corticosteroids pass through periods of acute stress, such as severe infections, trauma, a general anaesthetic or a major operation, the amount of corticosteroids must be increased forthwith. The actual increase will depend on the magnitude of the abnormal stress. In the case of an operation, for instance, it would be two or three or even four times, the daily dose. This raised dose must be maintained for from 36 to 48 hours and then be gradually reduced so that the previous level is attained in seven to ten days. If severe post-operation complications ensue, the high dose must be maintained for as long as necessary, since there is at this time a grave risk of collapse, from adrenal insufficiency.

When a patient who has had corticosteroids during the previous I 2 months, or even longer (especially a prolonged course, or high doses), has to undergo major surgery, he should be given the day before the operation and for two days subsequently the dose of corticosteroid that he had previously received. This amount should then be progressively reduced and eventually suppressed after 7 to io days.

Some surgeons advise that leprosy patients who have never had corticosteroids should receive them before undergoing major remedial surgery. There seems little scientifically based justification for this procedure.

SPECIAL PRESENTATIONS

Enteric-coated tablets of prednisolone are believed to withstand dissolution in the gastric juice, and hence minimize the risk of peptic ulceration (often symptomless) and its sequelae of perforation and haemorrhage.

Long-acting depot prednisolone, given parenterally, maintains an adequate blood level for as long as 7 days. When the patient's corticosteroid requirements are stable (as they often are, within narrow limits) and not subject to sudden fluctuations, there are obvious advantages in giving an injection weekly instead of an oral dose several times a day.

Soluble preparations are available which appear to be well tolerated by dyspeptic patients. Antacids should be prescribed on the slightest indication of epigastric discomfort or the passage of chemically detectable altered blood in the faeces.

CHANGE OF DRUG

Although there may be but slight differences in demonstrable potency and mode of action between proprietary brands of similar corticosteroids, it is a fact of observation that a change to another brand or the substitution of part of the maintenance dose of corticosteroid by an equivalent amount 
of a similar preparation, may be followed by obvious clinical and subjective improvement. Similarly, the temporary substitution of injectable cortisone for the whole or part of the dose of oral prednisolone may allow the physician to wean the long-term patient from dependence on the drug.

\section{LOCAL USES}

Although this article is concerned with the systemic use of corticosteroids, brief mention may be made in passing of their local uses, which in certain circumstances may result in a more obvious therapeutic action than that of the same drugs administered systemically.

I. In iritis, $0.25 \mathrm{ml}$. of an ophthalmic preparation of hydrocortisone in saline, introduced by means of. a fine needle under the conjunctiva latero-inferiorly after anaesthetization with a drop of 4 per cent cocaine solution, and repeated daily for several days, will relieve the pain and inflammation. 'The pupil is kept dilated with atropine in accordance with general principles. In the absence of facilities for subconjunctival introduction of hydrocortisone, hydrocortisone ophthalmic drops may be instilled twice daily into the lower sac.

2. Local infiltration of the tissues surrounding an acutcly inflamed peripheral nerve, with the following:

$$
\begin{array}{ll}
\text { Procain, I per cent } & 5 \mathrm{ml} \text {. } \\
\text { Hydrocortisone } & \mathrm{I} \mathrm{ml} \text {. } \\
\text { Hyaluronidase } & \mathrm{I} \mathrm{ml} \text {. }
\end{array}
$$

often affords relief lasting several days. 'The injection may be repeated.

3. Intralesional injection of $\mathrm{I} \mathrm{ml}$. of hydrocortisone suspension is of value in the pre-ulcerative phase of the acutely inflamed lepromatous infiltrated lesions of the skin. When ulceration has already occurred in such a lesion, the application of hydrocortisone ointment under an occlusive polythene dressing will usually ensure rapid healing and prevent severe scarring.

\section{(:ONTRA-INDICATIONS ANI DANGERS}

The absolute and relative contra-indications of corticosteroid therapy have been sufficiently stressed in the literature, e.g. tuberculosis (even quiescent), heart discase, peptic ulceration, hypertension, mental instability and severe infections. By reducing the beneficial inflammatory reaction to biological irritants, the drugs may mask serious infective states as well as immobolize the natural mechanisms of defence.

The dangers of high-dose and long-term corticosteroid therapy have also bcen stressed on numerous occasions - so much so, that advantage may not be taken of their real value in reducing harmful and excessive inflammatory response in reactional leprosy. As a rough and general rule, it may be said that the more deeply pigmented the patient, the less likely is he to suffer from the recognized serious side-effects of corticosteroid therapy, such as the following: peptic ulceration, diabetes, hyperpiesis, osteoporosis and vertebral collapse, pancreatic necrosis, lens opacities, 
jaundice, purpura, colitis; moon face, ocdema and cutancous striac are perhaps the commonest effects. The danger of retardation of growth in children on prolonged corticosteroid therapy is sufficiently important to be emphasized.

Early and unjustifiable recourse to corticosteroids is as unnecessary as it is potentially dangerous. Continuation of corticosteroid medication with no attempt at early weaning is to be condemned. Corticostcroids have no place in the prophylaxis of acute exaccrbation in leprosy.

\section{COOS T}

'The cost of corticosteroids unfortunately limits their use in many institutions, and may perhaps determine a less than adequate dosage for the individual patient. If the drugs are to be used at all, they must be given in adequate amounts and for the optimum duration to achicve the effect which they alone can assure.

However, it is possible to reduce the cost of treatment by using an (ffective brand of corticosteroid that produces, wcight for wcight, and shilling for shilling, a comparable therapeutic response. Prices vary locally within wide limits. A word of warning concerns unbranded products offered at ridiculously low prices: because they may contain variably low amounts of active product they may be almost without effect, and are too expensive - at any price - to use when a severely ill patient really necds a dependable drug available in a dependable concentration.

\section{S UMMAR Y}

Among the available adrenal corticosteroids and their synthetic analogues, prednisolone and betamethasone today hold pride of place in the control of acute exacerbation in lepromatous leprosy and of certain reactional phenomena in tuberculoid leprosy. Despite their recognized drawbacks and serious side-cffects, these drugs alone are effective in controlling severe exacerbation and permitting continuation of anti-leprosy therapy.

Detailed advice is given concerning the indications for corticosteroid treatment, the dosages that experience has found to be the best, the advisability and marner of discontinuing such treatment, etc. Bricf mention is made of ocular, perineural and intralesional use of hydrocortisone.

A CKNOWLEDGEMENTS

My thanks are duc to DR. S. O. Egwu ATu, Chief Medical Officer, Ministry of Health, Eastern Nigeria, for permission to publish this article. 